\title{
Chemical and biological detectors using ultra-high-Q microresonators
}

\author{
Andrea M. Armani, Kerry J. Vahala
}

Andrea M. Armani, Kerry J. Vahala, "Chemical and biological detectors using ultra-high-Q microresonators," Proc. SPIE 6376, Optomechatronic Micro/ Nano Devices and Components II, 637606 (18 October 2006); doi: $10.1117 / 12.691122$

SPIE. Event: Optics East 2006, 2006, Boston, Massachusetts, United States 


\title{
Chemical and Biological Detectors using Ultra-High-Q Microresonators Andrea M. Armani and Kerry J. Vahala \\ Department of Applied Physics, California Institute of Technology, 1200 E California Blvd, M/C 128-95, Pasadena, CA 91125 \\ armani@caltech.edu,vahala@caltech.edu
}

\begin{abstract}
Recently, a method for fabricating planar arrays of optical microtoroid resonators with quality factors greater than 500 million was developed. These devices have previously demonstrated Raman and OPO lasing and radiation pressure induced oscillations. When immersed in an aqueous environment, these devices are able to maintain their ultra-high Q factors by operating in the visible wavelength band, enabling very sensitive chemical and biological detection. The fabrication and optical properties of these devices will be described. These devices have performed both chemical and biological detection. Systems which have been detected include $\mathrm{D}_{2} \mathrm{O}$ in water and a variety of biological molecules. Sensitivity limits will also be discussed.
\end{abstract}

High Q and Ultra-high Q (UHQ) silica optical microcavities can perform as highly sensitive detectors [1-3]; their excellent transduction abilities is a result of the long photon lifetime within the whispering gallery of the microcavity. This signal amplification which is inherent to the device is unique among optical sensors. For example, in a waveguide sensor the photon interacts with the functionalized surface only once [4]; whereas the recirculation within the microcavity allows photons to interact with the surface many times, thus amplifying a single detection event. Additionally, the surface of silica-based microcavities is easily sensitized using silanization agents [5], amines, carbohydrates, the biotin-streptavidin system [6] or antibodies [7]. For example, silica microsphere resonators, with a properly sensitized surface, were recently used to distinguish between two strands of DNA [2]. Because the sensitivity increases as the quality factor increases, it is very important to maximize the $\mathrm{Q}$ of the cavity. In these previous experiments, the $\mathrm{Q}$ was limited to 1 million $\left(10^{6}\right)$ which is over 100 times lower than in air. If the $Q$ is increased to above 100 million, then label-free single molecule detection would be theoretically possible.[2]

There are many loss mechanisms which contribute to the quality factor of a resonant cavity, such as material loss, radiation loss, surface roughness loss, contamination loss and coupling loss.[8] While detailed studies have been performed to determine the limits of a resonator's quality factor in air [8], [9], [10], no comparable studies have been performed in water. Because most biological detection experiments are performed in a water-based solution, it is important to thoroughly understand the impact of this environment on resonator and optimize all parameters of the system (operational wavelength, resonator size, resonator material) to achieve the highest possible quality factor. By maximizing the $\mathrm{Q}$ of the cavity, the sensitivity of the cavity will also be optimized. 


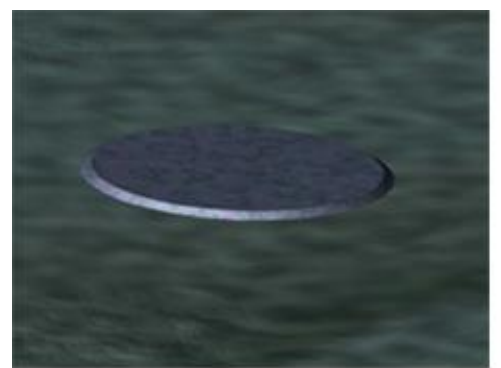

(a)

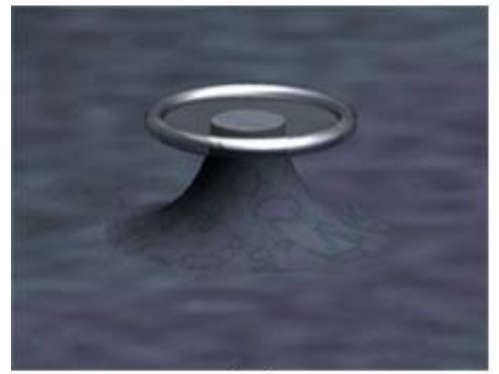

(c)

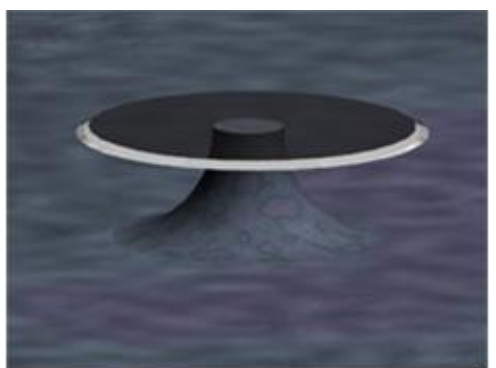

(b)

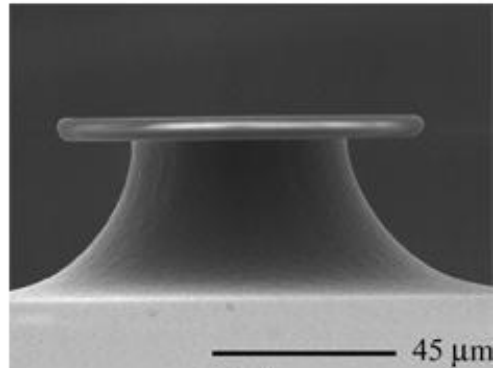

(d)

Figure 1: The fabrication of the ultra-high-Q microtoroid resonator consists of a single three step process. a) patterning the silica into an array of circular oxide pads, b) etching the silicon wafer substrate with $\mathrm{XeF}_{2}$ (an isotropic, gas-phase etchant) to form the high-Q microdisk, and c) reflowing the silica microdisk with a $\mathrm{CO}_{2}$ laser to form the toroidal resonator. d) Scanning electron micrograph of the UHQ microtoroid resonator.

To this end, UHQ silica microtoroid resonators were fabricated over a wide range (50250micron) of major toroid diameters using a previously outlined process (Figure 1). [11] Experiments were performed in both water $\left(\mathrm{H}_{2} \mathrm{O}\right)$ and deuterium oxide $\left(\mathrm{D}_{2} \mathrm{O}\right)$. The $\mathrm{D}_{2} \mathrm{O}$ was purchased from Aldrich. $\mathrm{D}_{2} \mathrm{O}$ was chosen as the second liquid because it has the same refractive index and, in turn, the same radiation-loss as $\mathrm{H}_{2} \mathrm{O}$. However, its absorption at all wavelengths tested is significantly less.[12] This contrast allowed for selective probing of the absorption-loss mechanism and verification of the model developed to describe this system.[13]

The model uses finite-element-analysis to predict the Q factor of microtoroid resonators immersed in water and accounted for two loss mechanisms: radiation-loss and absorptionloss. Both mechanisms are modified for aqueous versus air operation. In particular, the reduced refractive-index contrast for aqueous operation increases radiation loss at a fixed resonator radius, while water or $\mathrm{D}_{2} \mathrm{O}$ make absorption within the environment the central factor in limiting $\mathrm{Q}$ at large radii. It is important to note that the refractive index of water and $\mathrm{D}_{2} \mathrm{O}$ are identical, while the absorption of $\mathrm{D}_{2} \mathrm{O}$ is significantly less than water. This results in identical radiation-loss quality factors for both liquids but significantly higher absorption-loss quality factors for $\mathrm{D}_{2} \mathrm{O}$.

Measurements of the resonator quality factor and analysis of the modal structure were performed at three wavelength bands $(680,1300$ and $1500 \mathrm{~nm})$. By using these three wavelengths, a large range of the optical spectrum could be probed. Additionally, a large variation in optical absorption of either heavy water or water could be verified. For testing, a 
single-mode, tunable external cavity laser was coupled to a single-mode optical fiber containing a short, tapered section. Tapered fiber waveguides are high-efficiency probes of microcavities $[14,15]$. The tapered-fiber waveguides are fabricated by heating an optical fiber using an oxyhydric torch, while stretching the fiber. Tapered fibers for testing at $680 \mathrm{~nm}$ were pulled from F-SV fiber to an average waist diameter of $500 \mathrm{~nm}$. Tapers for testing at $1330 \mathrm{~nm}$ and $1550 \mathrm{~nm}$ were pulled from SMF fiber to an average diameter of 1 micron. The tapered section was used to couple power into the "whispering gallery modes" of the UHQ microtoroids.

During testing, the UHQ microtoroids were placed on a high-resolution translation stage (100-nm step resolution) and were monitored by two cameras (top and side view) simultaneously. The quality factor of the microtoroid resonator was first determined in air to ensure that it was above the theoretical limit for a given toroid diameter (once immersed in liquid). Then, with the taper waveguide in close proximity to the microtoroid, liquid was added and a cover slip was placed on top (Fig. 2). A "liquid" gap between the toroid and the taper was maintained when determining the quality factor in either $\mathrm{H}_{2} \mathrm{O}$ or $\mathrm{D}_{2} \mathrm{O}$ in order to maintain constant coupling between the microtoroid resonator and the taper waveguide.

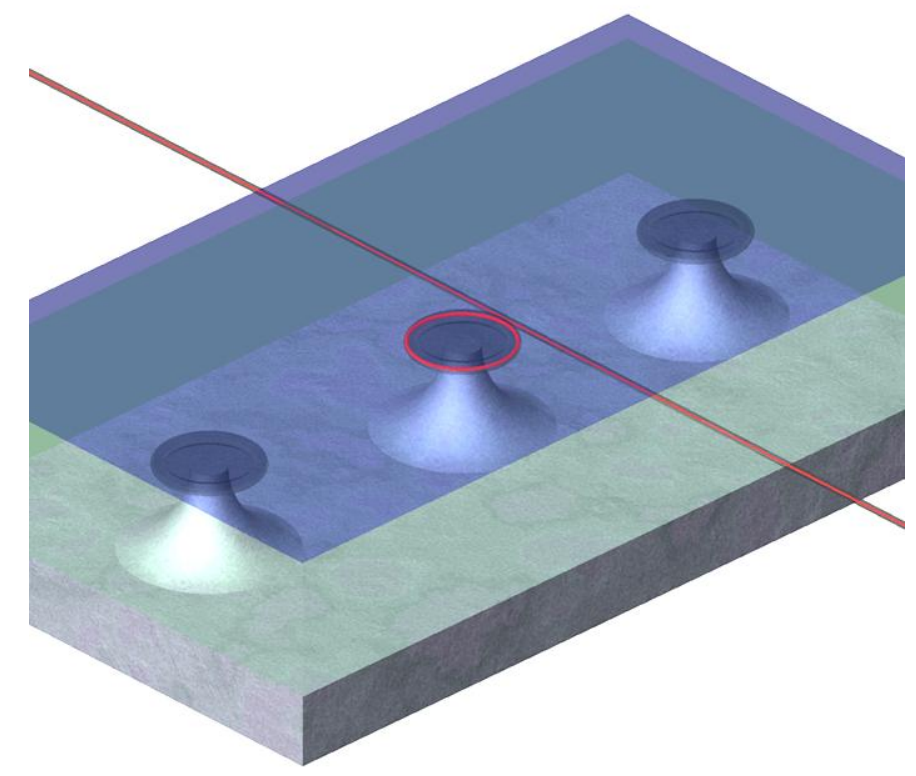

Figure 2. The Ultra-High-Q silica microtoroid is first coupled to the fiber taper waveguide and the $\mathrm{Q}$ is determined in air. After being immersed in either $\mathrm{H}_{2} \mathrm{O}$ or $\mathrm{D}_{2} \mathrm{O}$, a cover slip is placed on top forming a microaquarium.

Figure 3 shows typical transmission spectra in $\mathrm{H}_{2} \mathrm{O}$ at $1300 \mathrm{~nm}$ and in $\mathrm{D}_{2} \mathrm{O}$ at $1550 \mathrm{~nm}$. The spectra are taken in the under-coupled regime [14]. The modal structure is dominated by principal transmission minima, confirmed below, to be the fundamental transverse mode of the microtoroids. The intrinsic, Q factor (i.e., the Q factor in the absence of waveguide loading) was determined by scanning the single-mode laser (short-term linewidth of 300 $\mathrm{kHz}$ ) and measuring both the transmission and the loaded linewidth (full-width-halfmaximum) for several waveguide-resonator coupling conditions in the under-coupled regime. 
The intrinsic modal linewidth (and hence intrinsic Q) was then computed using a simple coupling model.[14] The laser scan frequency was optimized so as to ensure that neither scan direction (increasing frequency vs. decreasing frequency) nor scan frequency had any observable impact on linewidth.
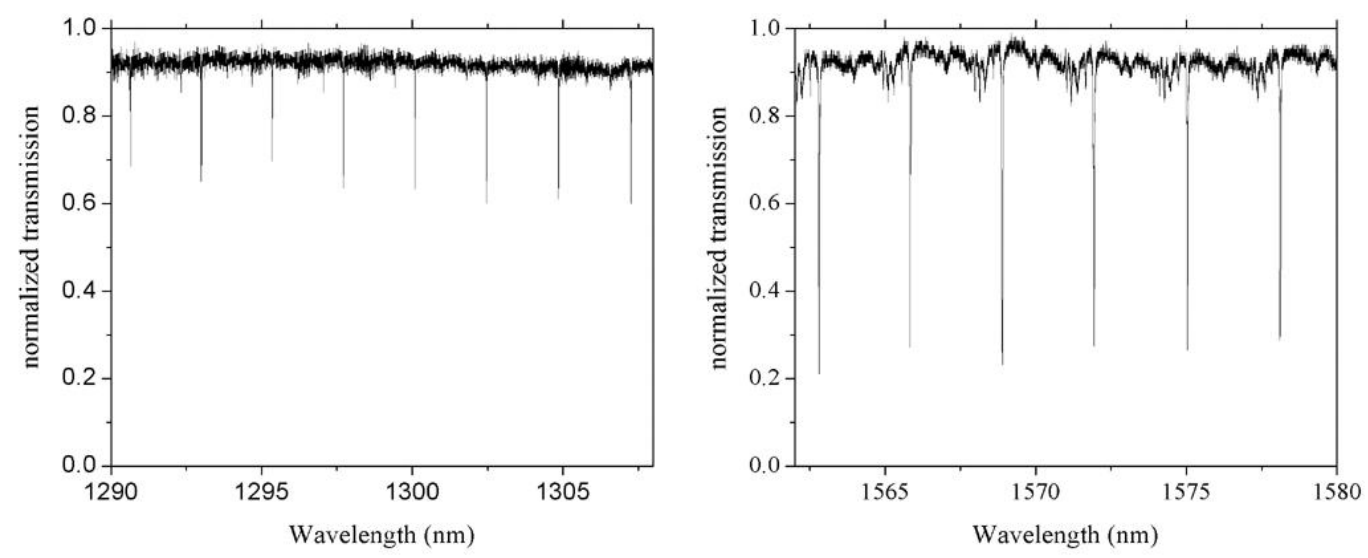

Figure 3. Transmission spectra of a microtoroid resonator in $\mathrm{D}_{2} \mathrm{O}$ at $1300 \mathrm{~nm}$ and $1550 \mathrm{~nm}$ bands. The resonator is highly under-coupled in the $1300 \mathrm{~nm}$ spectra. In the $1550 \mathrm{~nm}$ spectra, the resonator is also under-coupled but closer to being critically coupled.

The intrinsic $\mathrm{Q}$ factors measured in the $680 \mathrm{~nm}$ band plotted versus toroid major diameter are presented in Fig. 4 (triangular and circular points). Q factors trend to larger values with increasing toroid size. This behavior is in good agreement with predictions of the model (also shown in Fig. 4) and results from radiation loss. The maximum quality factor achieved in $\mathrm{H}_{2} \mathrm{O}$ was $2.3 \times 10^{8}$ and in $\mathrm{D}_{2} \mathrm{O}$ was $1.3 \times 10^{8}$. These values are notable as they represent the highest $\mathrm{Q}$ factors reported to date for operation in an aqueous environment. Measurements beyond Q factors of 500 million were not possible in this experiment owing to laser linewidth stability. In principle, however, larger toroid diameters should exhibit quality factors as high as $1 \times 10^{9}$, in water, and $1 \times 10^{10}$ in $\mathrm{D}_{2} \mathrm{O}$. 


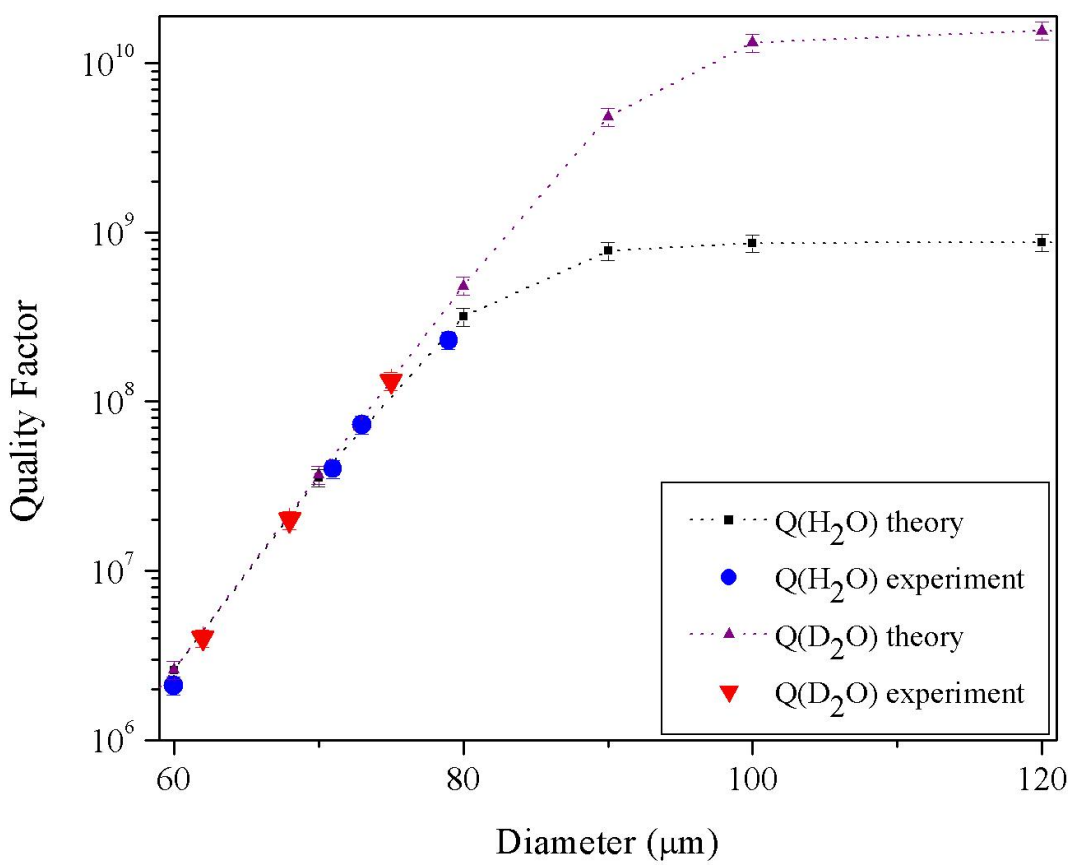

Figure 4. Quality factors measured and predicted in the $680 \mathrm{~nm}$ band plotted versus toroid major diameter. Q increases with major diameter over the range of diameters wherein radiation loss is the dominant loss mechanism. It then plateaus at values set by absorption of the aqueous environment. Above $5 \times 10^{8}$ data taking is unreliable due to laser-linewidth stability limitations. The maximum quality factor achieved in $\mathrm{H}_{2} \mathrm{O}$ was $2.3 \times 10^{8}$ and in $\mathrm{D}_{2} \mathrm{O}$ was $1.3 \times 10^{8}$.

The measured intrinsic $Q$ factors for microtoroids in $\mathrm{H}_{2} \mathrm{O}$ and $\mathrm{D}_{2} \mathrm{O}$ at different toroid diameters and measured in the $1300 \mathrm{~nm}$ band are plotted in Fig. 5. Both the radiation-losslimited regimes and the absorption-loss-limited regimes are clearly visible in these plots. Also plotted are predictions based on the model. Within this wavelength band, $\mathrm{D}_{2} \mathrm{O}$ has a lower optical absorption and hence exhibits an absorption-limited $\mathrm{Q}$ plateau that is significantly higher than for $\mathrm{H}_{2} \mathrm{O}$ (approximately $10^{6}$ for $\mathrm{H}_{2} \mathrm{O}$ versus above $10^{7}$ for $\mathrm{D}_{2} \mathrm{O}$ ). The origin of this absorption limit is the vibration overtone of water. In $\mathrm{D}_{2} \mathrm{O}$ this overtone is wavelength-shifted significantly, thereby increasing the observable $Q$ plateau. 


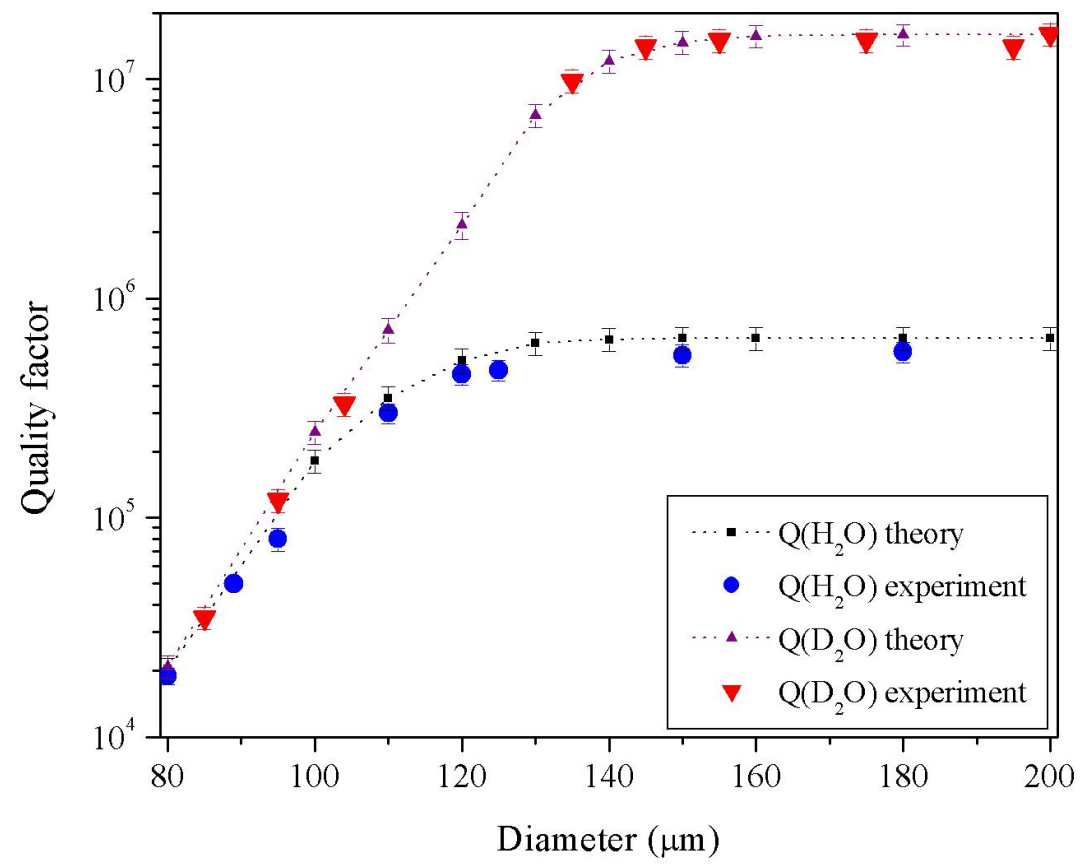

Figure 5. Quality factors measured and predicted in the $1300 \mathrm{~nm}$ wavelength band. Both the radiation-loss-limited (small toroid diameter) and aqueousabsorption-loss limited regimes (Q plateau) are apparent. The measured absorptive-loss limits are $5 \times 10^{5}$ (in $\mathrm{H}_{2} \mathrm{O}$ ) and $1.6 \times 10^{7}$ (in $\mathrm{D}_{2} \mathrm{O}$ ).

The measured intrinsic Q factors versus toroid diameter in the $1550 \mathrm{~nm}$ band are shown in Fig. 6, along with the predictions of the model. Again, there is good agreement between measurement and the model, showing the transition between the radiation-loss-limited and absorptive-loss-limited regimes. The strong $\mathrm{OH}$ overtone absorption in $\mathrm{H}_{2} \mathrm{O}$ lowers the $\mathrm{Q}$ plateau to $8 \times 10^{4}$, while for $\mathrm{D}_{2} \mathrm{O}$ the value is higher, increasing to above $3 \times 10^{6}$. 


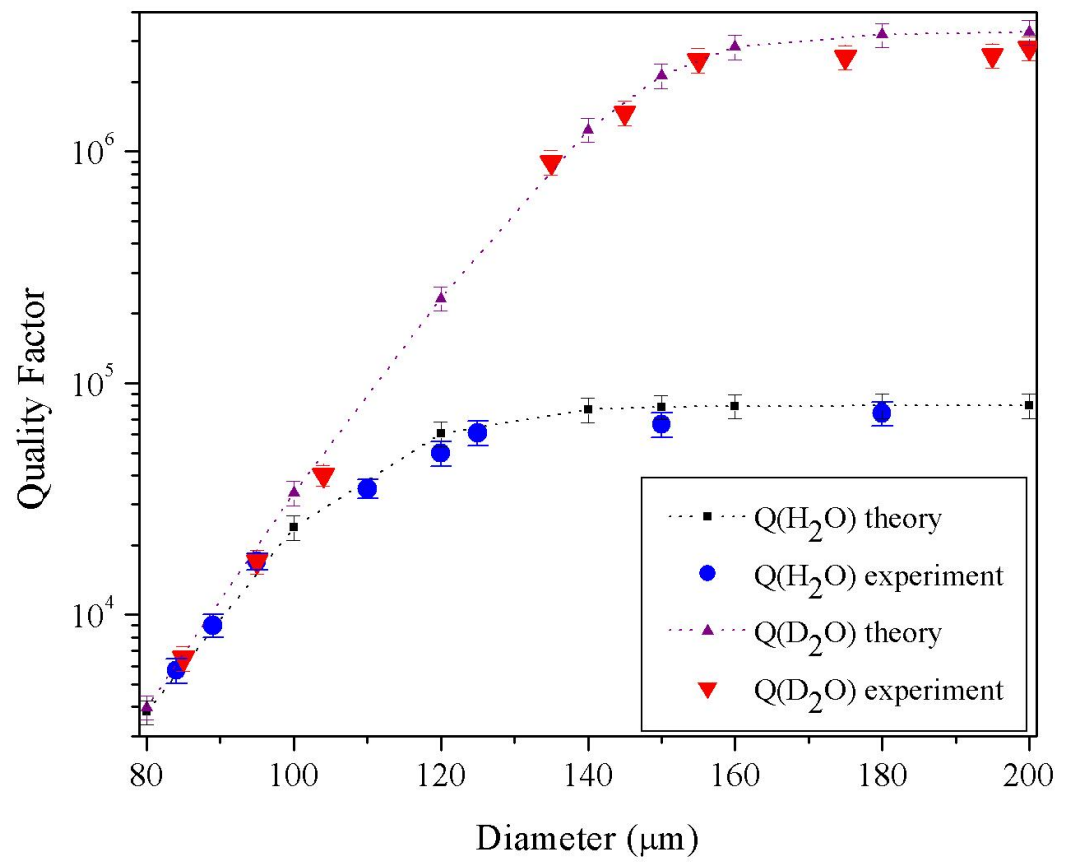

Figure 6. Quality factors measured and predicted in the $1550 \mathrm{~nm}$ band. In $\mathrm{H}_{2} \mathrm{O}$, the maximum quality factor achieved is $7 \times 10^{4}$. By changing to $\mathrm{D}_{2} \mathrm{O}$, the maximum quality factors increased to $2.8 \times 10^{6}$.

Having achieved one of the requirements for a highly sensitive resonant cavity sensor (ultrahigh-Q in water), a very simple yet elegant demonstration of this sensor's detection abilities presented itself in the form of heavy water detection.[16] At 1300nm, there is a large difference in the optical absorption of heavy water and water. This difference leads to a large change in the cavity $\mathrm{Q}$ depending on the percentage of heavy water in the environment around the toroid. Therefore, by monitoring the $\mathrm{Q}$, it is possible to determine how much heavy water is present in the water.

To demonstrate this effect, a simple testing procedure was designed: 1) immerse the microtoroid in $100 \% \mathrm{D}_{2} \mathrm{O}, 2$ ) gradually increase the concentration of $\mathrm{H}_{2} \mathrm{O}$ in $\mathrm{D}_{2} \mathrm{O}$, until $100 \%$ $\mathrm{H}_{2} \mathrm{O}$ is reached, and 3) return the concentration of $\mathrm{D}_{2} \mathrm{O}$ to $100 \%$ [16]. The difference between the quality factor in $\mathrm{H}_{2} \mathrm{O}$ and $\mathrm{D}_{2} \mathrm{O}$ is liquid-limited[13]; therefore, the quality factor can be described by: $\mathrm{Q}_{\text {liq }}=2 \pi \mathrm{n} / \lambda \alpha$, where $\mathrm{n}=$ effective refractive index, $\lambda=$ wavelength, and $\alpha$ is the absorption loss due to the liquid. The refractive index of $\mathrm{H}_{2} \mathrm{O}$ and $\mathrm{D}_{2} \mathrm{O}$ are similar and the resonant wavelength is constant.

During the initial series of measurements, the solutions were prepared in $10 \%$ increments $\left(10 \% \mathrm{H}_{2} \mathrm{O}\right.$ in $\mathrm{D}_{2} \mathrm{O}, 20 \% \mathrm{H}_{2} \mathrm{O}$ in $\mathrm{D}_{2} \mathrm{O}$, etc), starting with $100 \% \mathrm{D}_{2} \mathrm{O}$. After the quality factor was determined, all of the $\mathrm{D}_{2} \mathrm{O}$ was removed, and the chamber was then flushed five times with the next concentration solution (in this case, the $10 \% \mathrm{H}_{2} \mathrm{O}$ in $\mathrm{D}_{2} \mathrm{O}$ ), and the quality 
factor was again determined. This flushing process was followed for all solutions to remove trace amounts of higher or lower concentration solutions. As can be seen in Figure 7a, when the concentration of $\mathrm{D}_{2} \mathrm{O}$ was reduced, the quality factor decreased. The theoretical values for each concentration were calculated and are indicated by the dashed line. This $\mathrm{Q}$ decrease was reversible, and by increasing the $\mathrm{D}_{2} \mathrm{O}$ concentration, the quality factor is recovered.
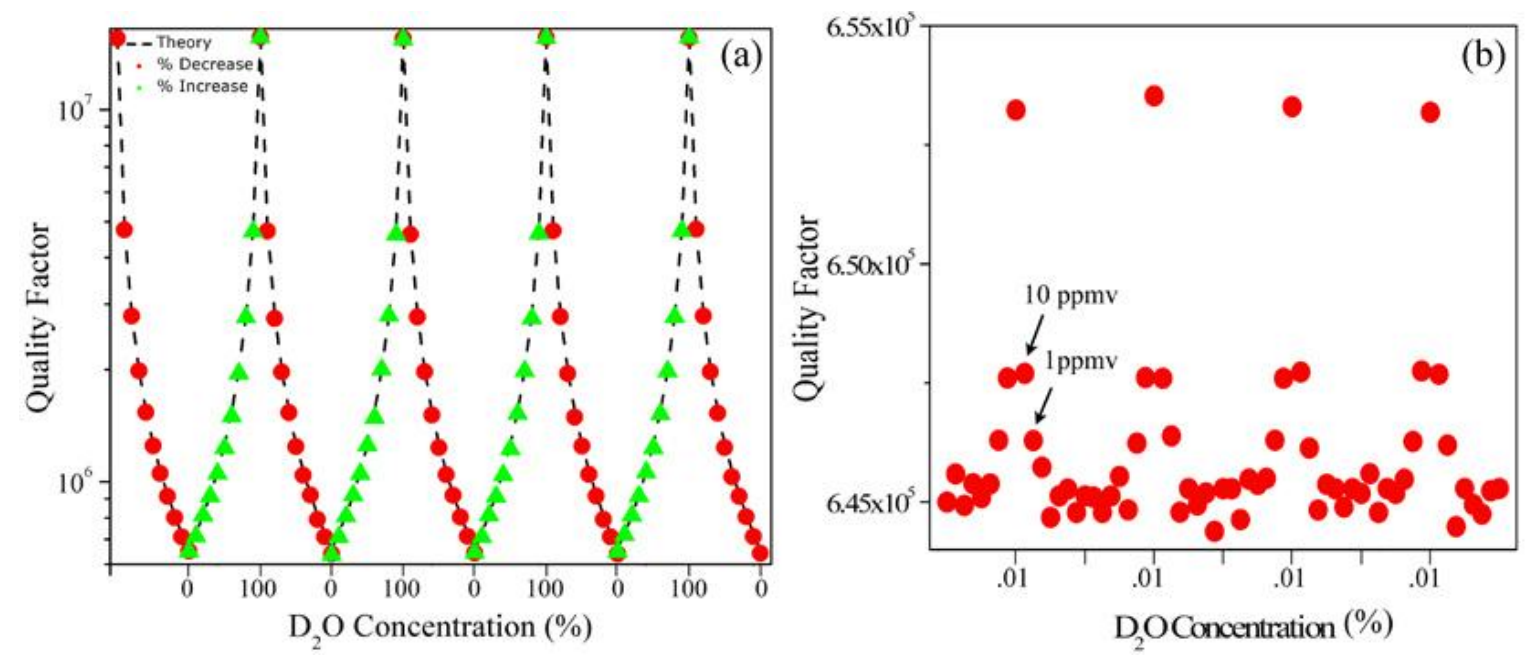

Figure. 7: a) The $\mathrm{Q}$ is systematically degraded (circles) and recovered (triangles) as the $\mathrm{D}_{2} \mathrm{O}$ and $\mathrm{H}_{2} \mathrm{O}$ are exchanged repeatedly. b) Starting with $100 \% \mathrm{H}_{2} \mathrm{O}$, the concentration of $\mathrm{D}_{2} \mathrm{O}$ was gradually increased using low concentration solutions ranging from $1 \times 10 \%$ to $.01 \%$. The minimum detectable change in $\mathrm{Q}$ was at $.0001 \%$ (1ppmv).

To determine the lower bound of the detection limit, larger dilutions of $\mathrm{D}_{2} \mathrm{O}$ in $\mathrm{H}_{2} \mathrm{O}$ were prepared, ranging from $.01 \%$ to $1 \times 10^{-9} \%$. As can be seen in Figure $7 \mathrm{~b}$, there is a strong signal at $.001 \% \mathrm{D}_{2} \mathrm{O}$ in $\mathrm{H}_{2} \mathrm{O}$ and a small, yet detectable, shift occurs with the $.0001 \% \mathrm{D}_{2} \mathrm{O}$ solution. These values are not believed to reflect the fundamental limit of the detection sensitivity of this device since no attempt was made to reduce operational sources of noise.

The ultra-high-Q microcavity has demonstrated the ability to detect the difference between two chemically similar species, $\mathrm{H}_{2} \mathrm{O}$ and $\mathrm{D}_{2} \mathrm{O}$, at low concentrations. This detection is based on the subtle difference in optical absorptions between $\mathrm{D}_{2} \mathrm{O}$ and $\mathrm{H}_{2} \mathrm{O}$, which is then magnified by the quality factor of the resonator. Using resonant cavities, it was possible to improve upon previous detection sensitivities (30ppmv) by over an order of magnitude.

In biological detection experiments, both specificity and sensitivity are important. While the ultra-high-Q optical resonator is inherently sensitive, as was demonstrated by the resonator's Q values $>10^{8}$, specificity is achieved by functionalizing the surface of the microtoroid. Several different surface functionalization techniques have been used (antibody, biotin), each one specific to the target molecule.

Both the biological detection and the heavy water detection experiments using the ultra-highQ toroidal resonators will be presented. Additionally, fundamental sensitivity limits will be discussed. 
References

[1] S. Blair and Y. Chen, "Resonant-enhanced evanescent-wave fluorescence biosensing with cylindrical optical cavities," Applied Optics, vol. 40, pp. 570-582, 2001.

[2] F. Vollmer, S. Arnold, D. Braun, I. Teraoka, and A. Libchaber, "Multiplexed DNA quanti. cation by spectroscopic shift of two microsphere cavities," Biophysical Journal, vol. 85, pp. 1974-1979, 2003.

[3] R. W. Boyd and J. E. Heebner, "Sensitive disk resonator photonic biosensor," Applied Optics, vol. 40, pp. 5742-5747, 2001.

[4] R. Horvath, H. C. Pedersen, N. Skivesen, D. Selmeczi, and N. B. Larsen, "Optical waveguide sensor for on-line monitoring of bacteria," Optics Letters, vol. 28, pp. 1233-1235, 2003.

[5] P. H. Maddox and D. Jenkins, "3-Aminopropyltriethoxysilane (Apes) - a New Advance in Section Adhesion," Journal of Clinical Pathology, vol. 40, pp. 12561257, 1987.

[6] L. A. Klumb, V. Chu, and P. S. Stayton, "Energetic roles of hydrogen bonds at the ureido oxygen binding pocket in the streptavidin-biotin complex," Biochemistry, vol. 37, pp. 7657-7663, 1998.

[7] R. A. Vijayendran and D. E. Leckband, "A quantitative assessment of heterogeneity for surface-immobilized proteins," Analytical Chemistry, vol. 73, pp. 471-480, 2001.

[8] M. L. Gorodetsky, A. A. Savchenkov, and V. S. Ilchenko, "Ultimate Q of optical microsphere resonators," Optics Letters, vol. 21, pp. 453-455, 1996.

[9] T. J. Kippenberg, S. M. Spillane, D. K. Armani, and K. J. Vahala, "Fabrication and coupling to planar high-Q silica disk microcavities," Applied Physics Letters, vol. 83, pp. 797-799, 2003.

[10] D. W. Vernooy, V. S. Ilchenko, H. Mabuchi, E. W. Streed, and H. J. Kimble, "HighQ measurements of fused-silica microspheres in the near infrared," Optics Letters, vol. 23, pp. 247-249, 1998.

[11] D. K. Armani, T. J. Kippenberg, S. M. Spillane, and K. J. Vahala, "Ultra-high-Q toroid microcavity on a chip," Nature, vol. 421, pp. 925-928, 2003.

[12] G. M. Hale and M. R. Querry, "Optical-Constants of Water in 200-Nm to 200-Mum Wavelength Region," Applied Optics, vol. 12, pp. 555-563, 1973.

[13] A. M. Armani, D. K. Armani, B. Min, K. J. Vahala, and S. M. Spillane, "Ultra-high-Q microcavity operation in $\mathrm{H} 2 \mathrm{O}$ and D2O," Applied Physics Letters, vol. 87, pp. -, 2005.

[14] M. Cai, O. Painter, and K. J. Vahala, "Observation of critical coupling in a fiber taper to a silica-microsphere whispering-gallery mode system," Physical Review Letters, vol. 85, pp. 74-77, 2000.

[15] S. M. Spillane, T. J. Kippenberg, O. J. Painter, and K. J. Vahala, "Ideality in a fibertaper-coupled microresonator system for application to cavity quantum electrodynamics," Physical Review Letters, vol. 91, pp. -, 2003. 
[16] A. M. Armani, Vahala, K. J., "Heavy water detection using ultra-high-Q microcavities," Optics Letters, vol. 31, 2006. 